\title{
Continuity Properties of the Representations of the Canonical Commutation Relations ${ }^{\star} \star \star$
}

\author{
E. J. Woods \\ Department of Mathematics, Queen's University, Kingston, Ontario, Canada
}

Received July 21, 1969; in revised form January 9, 1970

\begin{abstract}
We prove that a given representation of the canonical commutation relations can be extended uniquely by continuity to larger test function spaces which are maximal in the sense that no further extension is possible. For irreducible tensor product representations of the canonical commutation relations we give a necessary and a sufficient condition for the admissible test functions. We consider the problem of finding topologies on the test function spaces such that this extension can be obtained by a topological completion. Various examples are discussed.
\end{abstract}

\section{Introduction}

There is a variety of literature on the representations of the canonical commutation relations (hereafter referred to as CCRs) [1-3, 6-12, 14-22]. In the present paper we are concerned with the continuity properties of representations of the CCRs, and with topologies for the test function spaces. There is some discussion of this problem in the literature. Lemma 2.3 of Araki and Woods [2] gives a criterion for the continuity of the operators $U(f, g)$ and provides a method for extending by continuity a given representation to a larger class of test functions. However this lemma was stated somewhat ambiguously. In fact the present paper is essentially a clarification of this lemma. Streit [22] considered irreducible tensor product representations of the CCRs and gave a numerical criterion for a class of admissable test functions. Chaiken [6] constructed some representations of the CCRs which exhibit rather pathological continuity properties. After the present work was completed, the related results of Reid $[18,19]$ came to our attention. Recently Hegerfeldt and Klauder [12] have discussed the weakest vector topologies on $V_{\phi}, V_{\pi}$ such that the maps $f \rightarrow U(f), g \rightarrow V(g)$ are strongly continuous. A detailed discussion of the associated topologies on the test function spaces will be given in a paper with H. Araki [4].

* Supported in part by the National Research Council of Canada.

$\star \star$ An earlier version of the present work was distributed as a preprint entitled "Topologies for Test Function Spaces for Representations of the Canonical Commutation Relations". 
In Section 2 we give the definition of a representation of the CCRs $U(f, g)=e^{i \phi(f)} e^{i \pi(g)}, f \in V_{\phi}, g \in V_{\pi}$ over the test function spaces $V_{\phi}, V_{\pi}$ relative to a bilinear form $\langle f, g\rangle$ on $V_{\phi} \times V_{\pi}$. In Section 3 we prove some technical lemmas needed later (Lemma 3.5 seems to be a statement of independent interest). In Section 4 we discuss the extension by continuity of a given representation of the CCRs to larger test function spaces. The admissable test functions are given as appropriate equivalence classes of nets from the original test function spaces. In Section 5 we consider irreducible tensor product representations of the CCRs. We give a necessary condition and a sufficient condition for the admissable test functions. We show that these admissable test function spaces contain but in general do not coincide with the admissable test function spaces determined by Streit [22]. In Section 6 we discuss the problem of finding topologies $\tau_{\phi}, \tau_{\pi}$ on the test function spaces $V_{\phi}, V_{\pi}$ such that the spaces $\bar{V}_{\phi}, \bar{V}_{\pi}$ of admissable test functions are the completions of $V_{\phi}, V_{\pi}$ in the topologies $\tau_{\phi}, \tau_{\pi}$. In Section 7 we consider some representations of the CCRs (some of which are due to Chaiken [6]) which exhibit undesirable continuity properties. Our results do not provide a definitive treatment, and are probably best considered as a reformulation of the problem. In Section 8 we discuss briefly the questions considered above in the context of representations of topological groups which are not locally compact.

We use the following notation. If $\boldsymbol{A}$ is a set of bounded operators in a Hilbert space $H$, then $\boldsymbol{A}^{\prime}$ is the set of all bounded operators in $H$ which commute with every operator in the set $\boldsymbol{A}$. The algebra of all bounded operators in $H$ is denoted by $\boldsymbol{B}(H)$. The set of all unitary operators in $H$ is denoted by $\boldsymbol{U}(H)$. If $x_{\beta}, \beta \in B$ is a set of elements in a linear space $V$, their algebraic span is the subspace of all finite linear combinations $\sum c_{\beta_{j}} x_{\beta_{j}}$. We use $(x, y), x \in E, y \in F$ to denote the elements of the Cartesian product $E \times F$. We use $\langle x, y\rangle$ to denote a bilinear form on $E \times F$ where $E, F$ are linear spaces. We call the bilinear form non-degenerate if $E$ distinguishes points in $F$ and $F$ distinguishes points in $E$. For such paired spaces we denote the weak dual topology on $E$ by $w(E, F)$. If the topology $T$ is finer than the topology $S$ we write $T \geqq S$. If $T_{\beta}, \beta \in B$ is a collection of topologies for a space $X$, the supremum topology $\bigvee_{\beta \in B} T_{\beta}$ is the topology generated by the open sets for the $T_{\beta}, \beta \in B$.

\section{Definition of a Representation of the CCRs}

Definition 2.1. Let $V_{\phi}$ and $V_{\pi}$ be real vector spaces with a bilinear form $(f, g) \rightarrow\langle f, g\rangle$ from $V_{\phi} \times V_{\pi}$ into the field of real numbers. A representation of the Weyl commutation relations over $V_{\phi} \times V_{\pi}$ is the structure 
consisting of a complex Hilbert space $H$ and a map $(f, g) \rightarrow U(f, g)$ from $V_{\phi} \times V_{\pi}$ into $\boldsymbol{U}(H)$ such that

$$
U\left(f_{1}, g_{1}\right) U\left(f_{2}, g_{2}\right)=U\left(f_{1}+f_{2}, g_{1}+g_{2}\right) e^{i\left\langle f_{2}, g_{1}\right\rangle}
$$

If $V_{\phi}=V_{\pi}=V$ we call $U(f, g)$ a representation of the Weyl commutation relations over $V$. If the map $(s, t) \rightarrow U(s f, t g)$ is continuous from the usual topology for the real parameters $s, t$ to the strong operator topology for all $f, g$ we call $U(f, g)$ a continuous representation of the Weyl commutation relations. If in addition the bilinear form $\langle f, g\rangle$ is nondegenerate we call $U(f, g)$ a representation of the CCRs.

For some purposes the notation $U(f, g)=U(f) V(g)$ where

$$
\begin{gathered}
U(f)=U(f, 0), \\
V(g)=U(0, g)
\end{gathered}
$$

is more convenient. For continuous representations it follows from Stone's theorem that we can write $U(f)=e^{i \phi(f)}, V(g)=e^{i \pi(g)}$ where $\phi(f), \pi(g)$ are self adjoint. Definition 2.1 can be reformulated as a representation of the group of elements $(f, g, \sigma)$ where $f \in V_{\phi}, g \in V_{\pi}$ and $\sigma$ is a complex number of modulus one, with the multiplication law

$$
\left(f_{1}, g_{1}, \sigma_{1}\right)\left(f_{2}, g_{2}, \sigma_{2}\right)=\left(f_{1}+f_{2}, g_{1}+g_{2}, \sigma_{1} \sigma_{2} e^{i\left\langle f_{2}, g_{1}\right\rangle}\right)
$$

It should be noted that some of our terminology in definition 2.1 is new. The extension by continuity of a given representation of the CCRs can lead to degenerate bilinear forms [4], and it is therefor convenient to introduce a notation which allows for this.

\section{Some Technical Lemmas}

Lemma 3.1. Let $G$ be a group, let $H_{\alpha}$ be topological groups, let $f_{\alpha}$ be a representation of $G$ in $H_{\alpha}$, and let $\tau$ be the weakest topology on $G$ such that the maps $f_{\alpha}: G \rightarrow H_{\alpha}$ are continuous for all $\alpha$. Then $(G, \tau)$ is a topological group.

Proof. Let $\tau_{\alpha}$ be the weakest topology on $G$ such that the map $f_{\alpha}$ is continuous. Then $\tau=V \tau_{\alpha}$. Since the supremum of a family of topologies compatible with the group structure of $G$ is compatible with this structure ([5], Chapter III, page 102, problem 9), it is sufficient to prove that $\left(G, \tau_{\alpha}\right)$ is a topological group.

Given $x, y \in G$ and a neighbourhood $N$ of $x y^{-1}$ we must find neighbourhoods $N_{x}, N_{y}$ of $x, y$ such that $N_{x}\left(N_{y}\right)^{-1} \subset N$. Choose a neighbourhood $M$ of $f_{\alpha}\left(x y^{-1}\right)$ such that $f_{\alpha}^{-1}(M) \subset N$. Since $H_{\alpha}$ is a topological group there exist neighbourhoods $M_{x}, M_{y}$ of $f_{\alpha}(x), f_{\alpha}(y)$ such that 
$M_{x}\left(M_{y}\right)^{-1} \subset M$. It follows that $N_{x}=f_{\alpha}^{-1}\left(M_{x}\right), N_{y}=f_{\alpha}^{-1}\left(M_{y}\right)$ are the desired neighbourhoods. Q.E.D.

Lemma 3.2. The strong operator topology gives $\boldsymbol{U}(H)$ the structure of a topological group (the group operation is operator multiplication).

Proof. The sets $N(T, \phi, \varepsilon)=\{S:\|(S-T) \phi\|<\varepsilon\}$ for all $\phi \in H$ and all $\varepsilon>0$ are a basis for the neighbourhoods of $T$ in the strong operator topology. For $W_{1}, W_{2}, U_{1}, U_{2} \in \boldsymbol{U}(H)$ we have

$$
\begin{aligned}
& \left\|\left[W_{1} W_{2}-U_{1} U_{2}\right] \phi\right\|^{2}=2\left\{\|\phi\|^{2}-\operatorname{Re}\left(U_{1}^{-1} W_{1} W_{2} \phi, U_{2}\right)\right\} \\
& \quad=2\left\{\|\phi\|^{2}-\operatorname{Re}\left(\left[\left(U_{1}^{-1} W_{1}-1\right)+1\right] W_{2} \phi,\left[\left(U_{2}-W_{2}\right)+W_{2}\right] \phi\right)\right\} \\
& \quad=2\left\{\left(\left[U_{1}^{-1} W_{1}-1\right] W_{2} \phi, U_{2} \phi\right)+\left(W_{2} \phi,\left[U_{2}-W_{2}\right] \phi\right)\right\} .
\end{aligned}
$$

Now

$$
\left\|\left(U_{1}^{-1} W_{1}-1\right) \psi\right\|=\left\|\left(W_{1}-U_{1}\right) \psi\right\| .
$$

Thus $U_{1} \in N\left(W_{1}, W_{2} \phi, \frac{1}{4} \varepsilon^{2}\right)$ and $U_{2} \in N\left(W_{2}, \phi, \frac{1}{4} \varepsilon^{2}\right)$ implies that $U_{1} U_{2} \in N\left(W_{1} W_{2}, \phi, \varepsilon\|\phi\|^{-2}\right)$. Q.E.D.

Lemma 3.3. Let $\left\{U_{\alpha}\right\},\left\{V_{\alpha}\right\}$ be nets of unitary operators on a Hilbert space which converge to $U, V$ in the strong operator topology. Then $U_{\alpha} V_{\alpha} \rightarrow U V$ in the strong operator topology.

Proof. This is Lemma 2.2 of [2] which was stated for sequences but the proof given is valid without change for nets. Q.E.D.

Corollary 3.4. Let $U=$ strong $\lim U_{\alpha}, U_{\alpha}$ unitary. If $U_{\alpha}^{-1}$ is convergent then $U$ is unitary.

The following lemma, which seems to be of independent interest, will be used in Theorem 5.1. It was motivated by the following application. Let $\bigotimes_{n=1}^{\infty}\left(H_{n}, \Omega_{n}\right)$ be the complete tensor product space of von Neumann [17] and let $\otimes \phi_{n}$ be weakly equivalent to $\otimes \Omega_{n}$ (see Eq. (5.2)). Then there exist real numbers $x_{n}$ such that $\otimes e^{i x_{n}} \phi_{n}$ is strongly equivalent to $\otimes \Omega_{n}$. In an earlier version of this paper we proved that given any sequence $x_{n}$ of real numbers, there exist $\varepsilon_{k}>0, \sum \varepsilon_{k}<\infty$, integers $m_{k}$ and a partition of the positive integers into mutually disjoint finite sets $J_{k}$ such that

$$
\left|2 \pi m_{k}-\sum_{n \in J_{k}} x_{n}\right| \leqq \varepsilon_{k}
$$

It follows that $\bigotimes_{k=1}^{\infty}\left(\bigotimes_{n \in J_{k}} \phi_{n}\right) \in \bigotimes_{k=1}^{\infty}\left(\bigotimes_{n \in J_{k}}\left(H_{n}, \Omega_{n}\right)\right)$ which is canonically isomorphic to $\bigotimes_{k=1}^{\infty}\left(H_{n}, \Omega_{n}\right)$. H. Araki has provided a simplified proof, and an improvement of this result as follows. 
Lemma 3.5. Let $x_{n}, n=1,2, \ldots$ be a sequence of real numbers. Then there exist integers $m_{k}, N_{k}, k=1,2, \ldots$ where $N_{k}>N_{k-1}$ for all $k$ (where $\left.N_{0}=0\right)$ such that

$$
\sum_{k=1}^{\infty}\left|m_{k}-\sum_{n=N_{k-1}+1}^{N_{k}} x_{n}\right| \leqq \frac{1}{2}
$$

Proof. Let

$$
\begin{aligned}
U_{N} & =\sum_{n=1}^{N} x_{n}, \\
V_{N} & =U_{N}-\left[U_{N}\right],
\end{aligned}
$$

where $[x]$ is the greatest integer $q \leqq x$. Let $V$ be an accumulation point of the $V_{N}$. Then there exist integers $N_{k}, k=1,2, \ldots$ such that (i) $N_{k}>N_{k-1}$ for all $k$, (ii) $V_{N_{k}}$ is either monotonically increasing or decreasing to $V$, (iii) either $V_{N_{k}} \leqq \frac{1}{2}$ or $V_{N_{k}} \geqq \frac{1}{2}$ for all $k$, and (iv) $\left|\frac{1}{2}-V_{N_{1}}\right| \geqq \frac{3}{4}\left|\frac{1}{2}-V\right|$. Choose an integer $m_{1}$ so that

$$
\left|m_{1}-\sum_{n=1}^{N_{1}} x_{n}\right|=\inf \left(V_{N_{1}}, 1-V_{N_{1}}\right) .
$$

For $k=2,3, \ldots$ choose an integer $m_{k}$ so that

$$
\left|m_{k}-\sum_{n=N_{k-1}+1}^{N_{k}} x_{n}\right|=\left|V_{N_{k}}-V_{N_{k-1}}\right| \text {. }
$$

It follows that

$$
\sum_{k=1}^{\infty}\left|m_{k}-\sum_{n=N_{k-1}+1}^{N_{k}} x_{n}\right| \leqq \frac{1}{2}-\frac{1}{4}\left|\frac{1}{2}-V\right| .
$$

Q.E.D.

\section{The Extension of a Given Representation to Larger Test Function Spaces}

In this section we consider the extension by continuity of a given representation of the CCRs to larger test function spaces. We identify the admissable test functions as appropriate equivalence classes of nets from the original test function spaces. Since the extension can lead to degenerate bilinear forms [4], our definitions etc. are stated for continuous representations of the Weyl commutation relations (see Definition 2.1).

In considering the representations of infinite-dimensional Lie groups such as the CCRs, the question of comparing representations of different 
groups also becomes a natural one. Let $U_{F}(f, g)$ be the Fock representation of the CCRs over a real Hilbert space $V$. Then the map $f \rightarrow U_{F}(f)$ is continuous from the norm topology on $V$ to the strong operator topology [2]. Thus $\left\|f_{\alpha}-f\right\| \rightarrow 0$ implies that $U_{F}(f)=\operatorname{strong} \lim U_{F}\left(f_{\alpha}\right)$. It follows that $U_{F}(f, g)$ is determined by continuity by its restriction to any subspace $W$ of $V$ which is dense in the norm topology on $V$. More generally, if $U(f, g)$ is a representation of the CCRs over $V_{\phi} \times V_{\pi}$, one would expect that it is determined by continuity by its restriction to some subspaces $W_{\phi}, W_{\pi}$. In order to state this situation in an abstract way, we must be able to reconstruct the spaces $V_{\phi}, V_{\pi}$ from $W_{\phi}, W_{\pi}$. Actually one would like to do a little more. Namely given a representation of the CCRs, one would like to extend the representation by continuity to test function spaces which are maximal in the sense that no further extension by continuity is possible. The following definition will serve to characterize the admissable test functions.

Definition 4.1. Let $U(f, g)$ be a continuous representation of the Weyl commutation relations over $V_{\phi} \times V_{\pi}$. An admissable test function for the $U(f)$ is an equivalence class of nets $\left\{f_{\alpha}\right\}, f_{\alpha} \in V_{\phi}$ such that strong $\lim U\left(\lambda f_{\alpha}\right)$ exists for all real $\lambda$ and is strongly continuous in $\lambda$, the equivalence relation being given by $\left\{f_{\alpha}\right\} \sim\left\{f_{\alpha}^{\prime}\right\}$ if and only if strong $\lim U\left(\lambda f_{\alpha}\right)=$ strong $\lim U\left(\lambda f_{\alpha}^{\prime}\right)$ for all real $\alpha . V_{\phi}(U()$.$) denotes the space$ of all admissable test functions. Similarly one defines the admissable test functions for the $V(g)$ and $V_{\pi}(V()$.$) . If no ambiguity is possible we$ will write $\bar{V}_{\phi}, \bar{V}_{\pi}$ for $V_{\phi}(U()),. V_{\pi}(V()$.$) .$

Clearly $V_{\phi}, V_{\pi}$ can be canonically imbedded in $\bar{V}_{\phi}, \bar{V}_{\pi}$. If $\left\{f_{\alpha}\right\} \in \bar{V}_{\phi}$ (strictly speaking we should use the equivalence class of $\left\{f_{\alpha}\right\}$ ), it follows immediately from definition 4.1 that $\left\{\lambda f_{\alpha}\right\} \in \bar{V}_{\phi}$. If $\left\{f_{1 \alpha}\right\},\left\{f_{2 \alpha}\right\} \in \bar{V}_{\phi}$ it follows from Lemma 3.3 that $\left\{f_{1 \alpha}+f_{2 \alpha}\right\} \in \bar{V}_{\phi}$. Thus the equations

$$
\begin{gathered}
\lambda\left\{f_{\alpha}\right\}=\left\{\lambda f_{\alpha}\right\}, \\
\left\{f_{1 \alpha}\right\}+\left\{f_{2 \alpha}\right\}=\left\{f_{1 \alpha}+f_{2 \alpha}\right\}
\end{gathered}
$$

give $\bar{V}_{\phi}$ the structure of a real linear space.

Theorem 4.2. Let $U(f, g)$ be a continuous representation of the Weyl commutation relations over $V_{\phi} \times V_{\pi}$, and let $\bar{V}_{\phi}, \bar{V}_{\pi}$ be the spaces of admissable test functions. Then the bilinear form $\langle f, g\rangle$ on $V_{\phi} \times V_{\pi}$ can be extended uniquely by continuity to $\bar{V}_{\phi} \times \bar{V}_{\pi}$, and

$$
\bar{U}\left(\left\{f_{\alpha}\right\},\left\{g_{\alpha}\right\}\right)=\text { strong } \lim U\left(f_{\alpha}, g_{\alpha}\right)
$$

is a continuous representation of the Weyl commutation relations over $\bar{V}_{\phi} \times \bar{V}_{\pi}$. This extension is maximal in the sense that $\bar{V}_{\phi}(\bar{U}())=.\bar{V}_{\phi}$, $V_{\pi}(\bar{V}())=.\bar{V}_{\pi}$. 
Proof. We shall use the result that strong $\lim U\left(f_{\alpha}\right)=1$ implies that $\lim \left\langle f_{\alpha}, g\right\rangle=0$ for all $g \in V_{\pi}[4],[12]$.

We extend first the operators $U\left(\right.$.) to $\bar{V}_{\phi}$. Let $\left\{f_{\alpha}\right\} \in \bar{V}_{\phi}, \bar{U}\left(\lambda\left\{f_{\alpha}\right\}\right)$ = strong $\lim U\left(\lambda f_{\alpha}\right)$. Since $U\left(-f_{\alpha}\right)=U\left(f_{\alpha}\right)^{-1}$ it follows from Definition 4.1 and Lemma 3.3 that $\bar{U}\left(-\left\{f_{\alpha}\right\}\right)=\bar{U}\left(\left\{f_{\alpha}\right\}\right)^{-1}$. A second application of Lemma 3.3 gives strong $\lim U\left(f_{\alpha}-f_{\beta}\right)=1$ which implies that $\lim \left\langle f_{\alpha}-f_{\beta}, g\right\rangle=0$ for all $g \in V_{\pi}$. Thus $\left\langle f_{\alpha}, g\right\rangle$ is Cauchy and we can define

$$
\left\langle\left\{f_{\alpha}\right\}, g\right\rangle=\lim \left\langle f_{\alpha}, g\right\rangle
$$

If $\left\{f_{\alpha}\right\} \sim\left\{f_{\alpha}^{\prime}\right\}$ then strong $\lim U\left(f_{\alpha}-f_{\alpha}^{\prime}\right)=1$ which implies that $\lim \left\langle f_{\alpha}, g\right\rangle=\lim \left\langle f_{\alpha}^{\prime}, g\right\rangle$. Clearly Eq. (4.4) defines a bilinear form on $\bar{V}_{\phi} \times V_{\pi}$. We have

$$
\begin{aligned}
\bar{U}\left(\left\{f_{1 \alpha}\right\}\right) V(g) & \bar{U}\left(\left\{f_{2 \alpha}\right\}\right) \\
& =\left\{\text { strong } \lim U\left(f_{1 \alpha}\right)\right\} V(g)\left\{\text { strong } \lim U\left(f_{2 \alpha}\right)\right\} \\
& =\operatorname{strong} \lim U\left(f_{1 \alpha}\right) V(g) U\left(f_{2 \alpha}\right) \\
& =\operatorname{strong} \lim U\left(f_{1 \alpha}+f_{2 \alpha}\right) V(g) e^{i\left\langle f_{2 \alpha}, g\right\rangle} \\
& =\bar{U}\left(\left\{f_{1 \alpha}\right\}+\left\{f_{2 \alpha}\right\}\right) V(g) e^{i\left\langle\left\{f_{2 \alpha}\right\}, g\right\rangle}
\end{aligned}
$$

and $\bar{U}\left(\left\{f_{\alpha}\right\}\right) V(g)$ is a continuous representation of the Weyl commutation relations (the continuity follows directly from Definition 4.1). By a similar argument the operators $V\left(\right.$.) can now be extended to $\bar{V}_{\pi}$.

To prove that this extension is maximal, let $\left\{f_{\alpha \beta}\right\}, \beta \in B$ be elements of $\bar{V}_{\phi}$ such that strong $\lim _{\beta} \bar{U}\left(\lambda\left\{f_{\alpha \beta}\right\}\right)$ exists for all real $\lambda$ and is strongly continuous in $\lambda$. It follows from the iterated limits theorem ([14], page 69) that strong $\lim U\left(\lambda f_{\alpha \beta}\right)$ exists (where we take the product order on $(\alpha, \beta)$ ) and is equal to strong $\lim \bar{U}\left(\lambda\left\{f_{\alpha \beta}\right\}\right)$. Q.E.D.

Thus Definition 4.1 gives a natural definition of the admissable test functions, and Theorem 4.2 indicates that a given representation can be extended uniquely by continuity in a straightforward way to the admissable test functions. This situation occurs frequently in practise. For example, in quantum field theory one may construct representations of the CCRs where one takes $V_{\phi}=V_{\pi}$ to be some convenient space of functions $f(x), x \in R^{n}$ (such as the space $\mathscr{S}$ of Schwartz). However, it usually happens that the representation which one obtains can be extended to a larger class of test functions. In particular the Fock representation over an inner product space $V$ of test functions $f(x)$ is continuous in the norm topology and can thus be defined for all squareintegrable test functions [2].

Lemma 4.3. Let $U(f, g)$ be a continuous representation of the Weyl commutation relations over $V_{\phi} \times V_{\pi}$ on a separable Hilbert space $H$. If 
$f_{n}$ is a sequence in $V_{\phi}$ such that $U(\lambda)=$ strong $\lim U\left(\lambda f_{n}\right)$ exists for all real $\lambda$, then $U(\lambda)$ is strongly continuous in $\lambda$ and $\left\{f_{n}\right\} \in \bar{V}_{\phi}$.

Proof. Since the limit of a sequence of measurable functions is measurable, it follows that $U(\lambda)$ is weakly measurable in $\lambda$. On a separable Hilbert space this implies that $U(\lambda)$ is strongly continuous in $\lambda$.

Lemma 4.4. Let $U_{i}(f, g), i \in I$ be continuous representations of the Weyl commutation relations over $V_{\phi} \times V_{\pi}$, and let $\bar{V}_{\phi i}, \bar{V}_{\pi i}$ be the spaces of admissable test functions for $U_{i}(f, g)$. Then

$$
\begin{aligned}
& \bar{V}_{\phi}=\bigcap_{i \in I} \bar{V}_{\phi i}, \\
& \bar{V}_{\pi}=\bigcap_{i \in I} \bar{V}_{\pi i}
\end{aligned}
$$

are the spaces of admissable test functions for the representation $\bigoplus_{i \in I} U_{i}(f, g)$.

Proof. Follows immediately from the fact that for unitary operators we have strong $\lim \bigoplus_{i \in I} U_{i \alpha}=\bigoplus_{i \in I} U_{i}$ if and only if strong $\lim U_{i \alpha}=U_{i}$ for each $i \in I$. Q.E.D.

\section{Admissable Test Function Spaces for Tensor Product Representations of the CCRs}

As an illustration of the extension procedure given by Theorem 4.2 we consider irreducible tensor product representations of the CCRs $[14,22]$.

Let $V$ be an inner product space which is the algebraic span of an orthonormal basis $h_{n}, n=1,2, \ldots$ for $V$. For each $n$ let $U_{n}(s, t)$ be a representation of the CCRs for one degree of freedom defined in the Hilbert space $H_{n}$. Let $H_{\Omega}=\otimes\left(H_{n}, \Omega_{n}\right), \Omega_{n} \in H_{n},\left\|\Omega_{n}\right\|=1$ denote the incomplete tensor product space of von Neumann [17]. We call $\Omega=\otimes \Omega_{n}$ the reference vector. In the following we assume the reader is familiar with the results of $[14,22]$. Two product vectors $\otimes \beta_{n}, \otimes \gamma_{n}$ such that $0<\Pi\left\|\beta_{n}\right\|, \Pi\left\|\gamma_{n}\right\|<\infty$ are called strongly equivalent, $\otimes \beta_{n} \sim \otimes \gamma_{n}$, if

$$
\sum_{n}\left|1-\left(\beta_{n}, \gamma_{n}\right)\right|<\infty \text {. }
$$

They are called weakly equivalent, $\otimes \beta_{n} \stackrel{w}{\Perp} \otimes \gamma_{n}$, if

$$
\sum_{n}|1-|\left(\beta_{n}, \gamma_{n}\right) \|<\infty \text {. }
$$

For $f, g \in V$ we have $f=\sum f_{n} h_{n}, g=\sum g_{n} h_{n}$ where $f_{n}=g_{n}=0$ except for a finite number of $n$. The equation

$$
U_{\Omega}(f, g)=\otimes U_{n}\left(f_{n}, g_{n}\right)
$$


defines an irreducible tensor product representation of the CCRs over $V$ in the Hilbert space $H_{\Omega}$. Two such $U_{\beta}(f, g), U_{\gamma}(f, g)$ are unitarily equivalent if and only if the reference vectors $\otimes \beta_{n}, \otimes \gamma_{n}$ are weakly equivalent [14]. If no ambiguity is possible we write $U_{\Omega}(f, g)=U(f, g), H_{\Omega}=H$.

A product representation $U_{\Omega}(f, g)$ can always be extended by continuity to larger test function spaces which depend on the weak equivalence class of $\otimes \Omega_{n}$. According to Theorem 4.2 the spaces of admissable test functions are the spaces $V_{\phi}(\Omega), V_{\pi}(\Omega)$ obtained by considering all nets $f_{\alpha} \in V, g_{\alpha} \in V$ such that strong $\lim U\left(\lambda f_{\alpha}\right)$, strong $\lim V\left(\lambda g_{\alpha}\right)$ exist for all real $\lambda$ and are strongly continuous in $\lambda$. Since $\left\{f_{\alpha}\right\} \in V_{\phi}(\Omega)$ implies that $\lim \left\langle f_{\alpha}, g\right\rangle$ exists for all $g \in V$, it follows that there is a canonical map $\sigma$ from $V_{\phi}(\Omega)$ into the completion $V^{*}$ of $V$ in the topology $w(V, V)$ (in general the map $\sigma$ is many-to-one [4]). Thus if $\hat{f}=\left\{f_{\alpha}\right\} \in V_{\phi}(\Omega)$, $f_{\alpha} \in V_{b}$ then $f=\sigma \hat{f} \in V^{*}$ and $f=\lim f_{\alpha}\left(w\left(V^{*}, V\right) . V^{*}\right.$ is also the algebraic dual of $V$. We realize it as the linear space of all combinations $f=\sum f_{j} h_{j}$, $f_{j}$ real.

Theorem 5.1. Let $V, U_{\Omega}(f, g), \Omega=\otimes \Omega_{n}, V_{\phi}(\Omega), V_{\pi}(\Omega)$ be as above. A necessary condition that $f=\sum f_{n} h_{n} \in \sigma\left(V_{\phi}(\Omega)\right), g=\sum g_{n} h_{n} \in \sigma\left(V_{\pi}(\Omega)\right)$ is that $\otimes U_{n}\left(\lambda f_{n}\right) \Omega_{n} w \otimes \Omega_{n}, \otimes V_{n}\left(\lambda g_{n}\right) \Omega_{n} \stackrel{w}{\otimes} \otimes \Omega_{n}$ for all real $\lambda$. A sufficient condition is that there exist constants $\phi_{n}, \pi_{n}$ such that $\otimes U_{n}\left(\lambda f_{n}\right) \Omega_{n}$ $\sim \otimes e^{i \lambda \phi_{n}} \Omega_{n}, \otimes V_{n}\left(\lambda g_{n}\right) \Omega_{n} \sim \otimes e^{i \lambda \pi_{n}} \Omega_{n}$ for all real $\lambda$.

Proof. It is sufficient to consider $V_{\phi}$. Let $f=\sum f_{n} h_{n} \in \sigma\left(V_{\phi}(\Omega)\right)$. Then there exists a net $f(\alpha)=\sum f_{n}(\alpha) h_{n}, f(\alpha) \in V$ such that $\lim f(\alpha)=f\left(w\left(V^{*}, V\right)\right)$ and $\gamma(\lambda)=\lim U(\lambda f(\alpha)) \otimes \Omega_{n}$ exists and is not equal to zero for all real $\lambda$. One can use Definition 2.3 and Lemma 3.2 of [3] to prove that $\gamma(\lambda)$ is a product vector $\otimes \gamma_{n}(\lambda)$. Let $P_{n}(\lambda), Q_{n}(\lambda)$ be the orthogonal projections onto $U_{n}\left(\lambda f_{n}\right) \Omega_{n}, \gamma_{n}(\lambda)$. By considering $\gamma(\lambda)$ as a state on $\boldsymbol{B}\left(H_{\Omega}\right)$ and noting that $\lim f_{n}(\alpha)=f_{n}$ it is clear that we must have $Q_{n}(\lambda)=P_{n}(\lambda)$. Thus

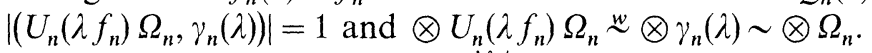

Now let $\otimes U_{n}\left(\lambda f_{n}\right) \Omega_{n} \sim \otimes e^{i \lambda \phi_{n}} \Omega_{n}$. By Lemma 3.5 there exist $\varepsilon_{k}>0$, $\sum \varepsilon_{k}<\infty$, integers $m_{k}$, and a partition of the positive integers into mutually disjoint finite sets $J_{k}$ such that

$$
\left|2 \pi m_{k}-\sum_{n \in J_{k}} \phi_{n}\right|<\varepsilon_{k}
$$

Using the associative law for tensor products we have $\bigotimes_{n=1}^{\infty}\left(H_{n}, \Omega_{n}\right)$ canonically isomorphic to $\bigotimes_{k=1}^{\infty}\left(\bigotimes_{n \in J_{k}} H_{n}, \bigotimes_{n \in J_{k}} \Omega_{n}\right)$. Now

$$
\bigotimes_{n \in J_{k}} e^{i \lambda \phi_{n}} \Omega_{n}=e^{i \delta_{k}} \bigotimes_{n \in J_{k}} \Omega_{n}
$$


where

$$
\sum\left|\delta_{k}\right|<\lambda \sum \varepsilon_{k}<\infty
$$

and thus

$$
\bigotimes_{k}\left(\bigotimes_{n \in J_{k}} e^{i \lambda \phi_{n}} \Omega_{n}\right) \sim \bigotimes_{k}\left(\bigotimes_{n \in J k} \Omega_{n}\right)
$$

which implies that

$$
\bigotimes_{k}\left(\bigotimes_{n \in J_{k}} U_{n}\left(\lambda f_{n}\right) \Omega_{n}\right) \sim \bigotimes_{k}\left(\bigotimes_{n \in J_{k}} \Omega_{n}\right)
$$

Let

$$
f(K)=\sum_{k=1}^{K}\left(\sum_{n \in J_{k}} f_{n} h_{n}\right)
$$

We now prove that strong $\lim U(\lambda f(K))$ exists for all real $\lambda$. Let $\gamma \in H$, and let $\varepsilon>0$. By Lemma 3.1 of [3] there exists $N<\infty$ and $\gamma_{N} \in \bigotimes_{n=1}^{N} H_{n}$ such that

$$
\left\|\gamma-\gamma_{N} \otimes\left(\bigotimes_{n=N+1}^{\infty} \Omega_{n}\right)\right\|<\varepsilon .
$$

Thus it is sufficient to prove that $\lim _{K \rightarrow \infty} U(\lambda f(K)) \otimes \Omega_{n}$ exists, and this follows from Eq. (5.5). It then follows from lemma 4.3 that $f \in \sigma\left(V_{\phi}(\Omega)\right)$. Q.E.D.

Corollary 5.2. Given $f=\sum f_{n} h_{n} \in V^{*}, f \notin V$, then there exists $\Omega=\otimes \Omega_{n}$ such that $f \notin \sigma(V(\Omega))$.

Proof. We have $f_{n} \neq 0$ for infinitely many $n$. Given $f_{n} \neq 0$ one can easily construct $\Omega_{n}$ such that $\left|\left(U_{n}\left(f_{n}\right) \Omega_{n}, \Omega_{n}\right)\right| \leqq 1-\delta$ for any $0<\delta \leqq 1$.

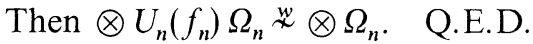

It is also a direct consequence of Theorem 6.3 that for any $\Omega=\otimes \Omega_{n}$, $V_{\phi}(\Omega), V_{\pi}(\Omega)$ are strictly larger than $V$ (this was also proved by Streit [22]).

We now consider the spaces of admissable test functions given by Streit [22]. Let $H^{\infty}=\otimes H_{n}$ denote the complete tensor product space of von Neumann [17]. For $f, g \in V^{*}$ the equations

$$
\begin{aligned}
W^{\infty}(f) \otimes \gamma_{n} & =\otimes U_{n}\left(f_{n}\right) \gamma_{n}, \\
X^{\infty}(g) \otimes \gamma_{n} & =\otimes V_{n}\left(g_{n}\right) \gamma_{n},
\end{aligned}
$$

define unitary operators $W^{\infty}(f), X^{\infty}(g)$ in $H^{\infty}$. In general the restrictions $W_{\Omega}(f), X_{\Omega}(g)$ of $W^{\infty}(f), X^{\infty}(g)$ to $H_{\Omega}$ are not unitary. Streit [22] gave the spaces of admissable test functions for the $U_{\Omega}(f), V_{\Omega}(g)$ to be

$$
\begin{aligned}
& V_{\phi}^{S}(\Omega)=\left\{f \in V^{*}: W_{\Omega}(\lambda f) \text { is unitary for all real } \lambda\right\}, \\
& V_{\pi}^{S}(\Omega)=\left\{g \in V^{*}: X_{\Omega}(\lambda g) \quad \text { is unitary for all real } \lambda\right\} \text {. }
\end{aligned}
$$


It follows immediately that

$$
\begin{array}{ll}
V_{\phi}^{S}(\Omega)=\left\{f=\sum f_{n} h_{n}: \otimes U_{n}\left(\lambda f_{n}\right) \Omega_{n} \sim \otimes \Omega_{n}\right. & \text { for all real } \lambda\}, \\
V_{\pi}^{S}(\Omega)=\left\{g=\sum g_{n} h_{n}: \otimes V_{n}\left(\lambda g_{n}\right) \Omega_{n} \sim \otimes \Omega_{n}\right. & \text { for all real } \lambda\} .
\end{array}
$$

Theorem 5.1 then gives $V_{\phi}^{S}(\Omega) \subset \sigma\left(V_{\phi}(\Omega)\right), V_{\pi}^{S}(\Omega) \subset \sigma\left(V_{\pi}(\Omega)\right)$. One can easily show that $f \in V_{\phi}^{S}(\Omega)$ if and only if $U(\lambda f)=$ strong $\lim U\left(\lambda f^{(n)}\right)$ where $f^{(n)}$ is the truncated test function $f^{(n)}=\sum_{j=1}^{n} f_{j} h_{j}$. Thus for some tensor product representations (e.g. the Fock representation) we have $V_{\phi}^{S}(\Omega)=\sigma\left(V_{\phi}(\Omega)\right)=V_{\phi}(\Omega), V_{\pi}^{S}(\Omega)=\sigma\left(V_{\pi}(\Omega)\right)=V_{\pi}(\Omega)$.

Lemma 5.3. There exists $\Omega=\otimes \Omega_{n}$ such that $V_{\phi}^{S}(\Omega) \neq \sigma\left(V_{\phi}(\Omega)\right)$.

Proof. Let

$$
\Omega_{n}(x)=\left(\sigma_{n} / \pi\right)^{1 / 4} e^{-\sigma_{n}(x-\varepsilon(n))^{2} / 2}
$$

where

$$
\varepsilon(n)= \begin{cases}+1, & n \text { even } \\ -1, & n \text { odd }\end{cases}
$$

and

$$
\sigma_{n}=n^{2} .
$$

Then $\left\|\Omega_{n}\right\|=1$. Consider $F=\sum F_{n} h_{n}$ where $F_{n}=1$ for all $n$. We have

$$
\left\|U_{n}(\lambda) \Omega_{n}-e^{i \varepsilon(n) \lambda} \Omega_{n}\right\|=\frac{1}{2} \lambda / \sigma_{n}+0\left(\lambda^{2} / \sigma_{n}^{2}\right) .
$$

It follows from Eq. (5.1) that $\sum\left\|\beta_{n}-\gamma_{n}\right\|<\infty$ implies that $\otimes \beta_{n} \sim \otimes \gamma_{n}$. Thus Eqs. (5.13), (5.14) imply that $\otimes U_{n}(\lambda) \Omega_{n} \sim \otimes e^{i \varepsilon(n) \lambda} \Omega_{n} \nsim \otimes \Omega_{n}$. Thus $F \notin V_{\phi}^{S}(\Omega)$. But it follows from Theorem 5.1 that $F \in \sigma\left(V_{\phi}(\Omega)\right)$. Q.E.D.

It might be remarked that the criterion of Streit is not a natural one. Let $F, \Omega$ be as in the proof of Lemma 5.3. If one considers $U_{\Omega}(f, g)$ as the tensor product $\bigoplus_{n=1}^{\infty}\left(U_{2 n}\left(f_{2 n}, g_{2 n}\right) \otimes U_{2 n-1}\left(f_{2 n-1}, g_{2 n-1}\right)\right)$ then $F$ would be an admissable test function according to the criterion of Streit.

\section{Topologies on the Test Function Spaces Induced by a Representation of the CCRs}

The extension procedure given by Theorem 4.2 is closely related to the following well-known result ([5], Chap. III, Sec. 3, Proposition 5). Let $G_{1}$ be a topological group, $G_{2}$ a complete Hausdorff topological group, and let $H_{1}, H_{2}$ be everywhere dense subgroups of $G_{1}, G_{2}$. Then 
every continuous representation of $H_{1}$ into $H_{2}$ can be uniquely extended to a continuous representation of $G_{1}$ into $G_{2}$. In this section we consider the problem of finding, for a given representation of the CCRs, topo$\operatorname{logies} \tau_{\phi}, \tau_{\pi}$ for the test function spaces such that this completion procedure gives the extension to the admissable test functions. Unfortunately there are some difficulties associated with the requirement that $U(s f, t g)$ be strongly continuous in the real parameters $s, t$ which have not yet been resolved, so that we do not provide a definitive solution of this problem.

We want a topology $\tau_{\phi}$ on $V_{\phi}$ such that

(i) the map $f \rightarrow U(f)$ is strongly continuous,

(ii) the completion $\bar{V}_{\phi}\left(\tau_{\phi}\right)$ of $V$ is a vector space (with $V_{\phi}$ as a dense subspace),

(iii) $\bar{V}_{\phi}\left(\tau_{\phi}\right)$ is canonically isomorphic to the space $\bar{V}_{\phi}$ of admissable test functions.

It follows from (ii) that $f_{\alpha}$ is a Cauchy net in $V_{\phi}$ if and only if $\lambda f_{\alpha}$ is a Cauchy net for all real $\lambda$. Since a map is continuous if and only if it carries convergent nets into convergent nets, it follows that the map $f \rightarrow \lambda f$ must be continuous.

It now follows from (i) that the maps $f \rightarrow U(\lambda f)$ must be continuous for all real $\lambda$. If we take $\tau_{\phi}$ to be the weakest topology on $V_{\phi}$ such that the maps $f \rightarrow U(\lambda f)$ are strongly continuous for all real $\lambda$, then $V_{\phi}$ can be canonically imbedded in $\bar{V}_{\phi}\left(\tau_{\phi}\right)$. However we have been unable to prove that $U(\lambda f)$ is strongly continuous in $\lambda$ for all $f \in \bar{V}_{\phi}\left(\tau_{\phi}\right)$. On the other hand, let $\tau_{\phi V}$ be the weakest vector topology on $V_{\phi}$ such that the map $f \rightarrow U(f)$ is strongly continuous $[4,12]$. Clearly $\tau_{\phi V} \geqq \tau_{\phi}$, and $U(\lambda f)$ is strongly continuous in $\lambda$ for all $f \in \bar{V}_{\phi}\left(\tau_{\phi V}\right)$. However in general $\tau_{\phi V}$ is strictly finer than $\tau_{\phi}[4]$, and we have been unable to prove that $\bar{V}_{\phi}\left(\tau_{\phi V}\right)$ contains all the admissable test functions. Now it is clear from the proof of Theorem 4.2 that $U(f, g), f \in \bar{V}_{\phi}\left(\tau_{\phi}\right), g \in V_{\pi}$ satisfy the Weyl commutation relations.

Note added in proof. H. Araki has given an example of the form $U(f, g)=e^{i T(f)} U_{F}(f, g)$ where $T(f)$ is a linear map into $R$ (the real line) such that $U(\lambda f)$ is not strongly continuous for some $f \in \bar{V}_{\phi}\left(\tau_{\phi}\right)$.

Definition 6.1. Let $U(f, g)$ be a continuous representation of the Weyl commutation relations over $V_{\phi} \times V_{\pi}$. The topologies $\tau_{\phi}, \tau_{\pi}$ induced on $V_{\phi}, V_{\pi}$ by the representation $U(f, g)$ are the weakest topologies on $V_{\phi}, V_{\pi}$ such that the maps $f \rightarrow U(\lambda f), g \rightarrow V(\lambda g)$ are strongly continuous for all real $\lambda$.

Lemma 6.2. Let $U(f, g)$ be a representation of the CCRs over $V_{\phi} \times V_{\pi}$, and let $\tau_{\phi}$ be the induced topology on $V_{\phi}$. Then $\left(V_{\phi}, \tau_{\phi}\right)$ is a topological group under addition. 
Proof. Follows immediately from Lemma 3.2, Lemma 3.1, and Definition 6.1. Q.E.D.

Lemma 6.3. Let $U(f, g)$ be a representation of the CCRs over $V_{\phi} \times V_{\pi}$, and let $\bar{V}_{\phi}$ be the completion of $V_{\phi}$ in the induced topology $\tau_{\phi}$. If $f=\lim f_{\alpha}$, $f \in \bar{V}_{\phi}, f_{\alpha} \in V_{\phi}$ then

$$
\lambda f=\lim \lambda f_{\alpha}
$$

gives $\bar{V}_{\phi}$ the structure of a vector space.

Proof. Clearly $\bar{V}_{\phi}$ is an abelian group under addition. It follows from definition 6.1 that $f_{\alpha}$ is a Cauchy net if and only if $\lambda f_{\alpha}$ is a Cauchy net for all real $\lambda$. It follows immediately from Eq. (6.1) that the axioms for scalar multiplication are satisfied. Q.E.D.

Theorem 6.4. Let $U(f, g)$ be a representation of the CCRs over $V_{\phi} \times V_{\pi}$ on a Hilbert space $H$, and let $W_{\phi}, W_{\pi}$ be subspaces of $V_{\phi}, V_{\pi}$ which are dense in the topologies $\tau_{\phi}, \tau_{\pi}$ induced by the representation $U(f, g)$. Let $\left(\bar{W}_{\phi}, \tau_{\phi}\right),\left(\bar{W}_{\pi}, \tau_{\pi}\right)$ be the complete topological groups obtained by completing $\left(W_{\phi}, \tau_{\phi}\right),\left(W_{\pi}, \tau_{\pi}\right)$. Then the bilinear form $\langle f, g\rangle$ on $W_{\phi} \times W_{\pi}$ can be extended uniquely by continuity to a bilinear form on $\bar{W}_{\phi} \times \bar{W}_{\pi}$, and the representation $U(f, g)$ can be extended uniquely by continuity to a representation $\bar{U}(f, g)$ of the Weyl commutation relations over $\bar{W}_{\phi} \times \bar{W}_{\pi}$ such that $\bar{U}(f, g)=U(f, g), f \in V_{\phi}, g \in V_{\pi}$ (where we identify $V_{\phi}, V_{\pi}$ as dense subspaces of $\left.\bar{W}_{\phi}, \bar{W}_{\pi}\right)$. The topologies on $W_{\phi}, W_{\pi}$ induced by the representation $U(f, g)$ coincide with the topologies $\tau_{\phi}, \tau_{\pi}$.

Proof. The proof is virtually identical to the proof of Theorem 4.2 (except that the condition of strong continuity in $\lambda$ is absent here). Q.E.D.

Lemma 6.5. Let $U(f, g)$ be a representation of the CCRs over $V_{\phi} \times V_{\pi}$ which is a discrete direct sum of the representations $U_{i}(f, g), i \in I$. Let $\tau_{\phi}, \tau_{\phi}^{i}$ and $\tau_{\pi}, \tau_{\pi}^{i}$ be the topologies on $V_{\phi}$ and $V_{\pi}$ induced by the representations $U(f, g), U_{i}(f, g), i \in I$. Then

$$
\tau_{\phi}=\bigvee_{i \in I} \tau_{\phi}^{i} \text { and } \tau_{\pi}=\bigvee_{i \in I} \tau_{\pi}^{i}
$$

Proof. We have $U(f, g)=\oplus U_{i}(f, g)$. Clearly the maps $f \rightarrow U(\lambda f)$ are strongly continuous if and only if the maps $f \rightarrow U_{i}(\lambda f)$ are strongly continuous for all $i \in I$. Q.E.D.

For some representations the continuity of $f \rightarrow U(\lambda f)$ follows from the continuity of $f \rightarrow U(f)$, but this is not true in general. In fact it is not even sufficient to require that $f \rightarrow U(\lambda f)$ be continuous for a countable number of $\lambda[4]$. 


\section{Some Examples}

The following example is a trivial modification of one given by Chaiken [6]. Let $U(f, g)$ be a representation of the CCRs over $V_{\phi} \times V_{\pi}$. Let $)_{\phi}, T_{\pi}$ be linear maps from $V_{\phi}, V_{\pi}$ into $R$ (the real line). Then

$$
U_{1}(f, g)=e^{i T_{\phi}(f)} e^{i T_{\pi}(g)} U(f, g)
$$

is also a representation of the CCRs over $V_{\phi} \times V_{\pi}$. That $U_{1}(f, g)$ satisfies the Weyl commutation relation follows immediately from the linearity of $T_{\phi}$ and $T_{\pi}$, and the fact that $U(f, g)$ satisfies the Weyl commutation relation. Since $T_{\phi}(s f)=s T_{\phi}(f), T_{\pi}(t g)=t T_{\pi}(g)$, it follows that $U_{1}(s f, t g)$ is strongly continuous in the real variables $s$ and $t$ for all $f \in V_{\phi}, g \in V_{\pi}$. Now let $W_{\phi}, W_{\pi}$ be proper subspaces of $V_{\phi}, V_{\pi}$ which are dense in the topologies $w\left(V_{\phi}, V_{\pi}\right), w\left(V_{\pi}, V_{\phi}\right)$ respectively. The essential point is that there exist $T_{\phi}, T_{\pi}$ which are zero on $W_{\phi}, W_{\pi}$ but do not vanish identically. In fact, as the following construction shows, there is a vast number of such $T_{\phi}, T_{\pi}$ (provided $V_{\phi}-W_{\phi}$ and $V_{\pi}-W_{\pi}$ are not too small). Let $\left\{h_{\alpha}^{\phi}, \alpha \in A_{\phi}\right\},\left\{h_{\alpha}^{\pi}, \alpha \in A_{\pi}\right\}$ be algebraic bases for $W_{\phi}, W_{\pi}$ and extend them to algebraic bases $\left\{h_{\alpha}^{\phi}, \alpha \in A_{\phi} ; e_{\beta}^{\phi}, \beta \in B_{\phi}\right\},\left\{h_{\alpha}^{\pi}, \alpha \in A_{\pi} ; e_{\beta}^{\pi}, \beta \in B_{\pi}\right\}$ for $V_{\phi}, V_{\pi}$. Let $\left\{x_{\beta}, \beta \in B_{\phi}\right\},\left\{y_{\beta}, \beta \in B_{\pi}\right\}$ be arbitrary sets of real numbers (not all zero). Define

$$
\begin{array}{ll}
T_{\varphi}\left(h_{\alpha}^{\varphi}\right)=0 & \alpha \in A_{\phi}, \\
T_{\varphi}\left(e_{\beta}^{\varphi}\right)=x_{\beta} & \beta \in B_{\phi}, \\
T_{\pi}\left(h_{\alpha}^{\pi}\right)=0 & \alpha \in A_{\pi}, \\
T_{\pi}\left(e_{\beta}^{\pi}\right)=y_{\beta} & \beta \in B_{\pi} .
\end{array}
$$

Then $U_{1}(f, g)$ is a representation of the CCRs which coincides with $U(f, g)$ for $f \in W_{\phi}, g \in W_{\pi}$ but not for all $f \in V_{\phi}, g \in \mathrm{V}_{\pi}$.

The following theorem is a straightforward generalization of Propositions 5.1 and 5.2 of [6].

Theorem 7.1. Let $U(f, g)$ be a representation of the CCRs over $V_{\phi} \times V_{\pi}$. Let $W_{\phi}, W_{\pi}$ be subspaces of $V_{\phi}, V_{\pi}$ such that $\left\{U(f, g) ; f \in W_{\phi}, g \in W_{\pi}\right\}^{\prime \prime}$ $=\left\{U(f, g) ; f \in V_{\phi}, g \in V_{\pi}\right\}^{\prime \prime}$. Then $U_{1}(f, g)=X(f, g) U(f, g)$ is a representation of the CCRs over $V_{\phi} \times V_{\pi}$ such that $U_{1}(f, g)=U(f, g), f \in W_{\varphi}, g \in W_{\pi}$ if and only if

(i) $X(f, g)$ is unitary,

(ii) $X(f, g)=1, f \in W_{\phi}, g \in W_{\pi}$,

(iii) $X(s f, t g)$ is strongly continuous in the real variables $s$ and $t$ for all $f \in V_{\phi}, g \in V_{\pi}$,

(iv) $X(f, g) \in\left\{U(f, g) ; f \in V_{\phi}, g \in V_{\pi}\right\}^{\prime}$,

(v) $X\left(f_{1}, g_{1}\right) X\left(f_{2}, g_{2}\right)=X\left(f_{1}+f_{2}, g_{1}+g_{2}\right)$. 
Furthermore, $U_{1}(f, g)$ is unitarily equivalent to $U(f, g)$ if and only if $X(f, g)=1$ for all $f \in V_{\phi} . g \in V_{\pi}$.

Proof. If $X(f, g)$ satisfies (i) - (v) then $X(f, g) U(f, g)$ is clearly a representation of the CCRs which coincides with $U(f, g)$ on $W_{\phi} \times W_{\pi}$. Conversely, assume that $U_{1}(f, g)=X(f, g) U(f, g)$ is such a representation. Clearly $X(f, g)$ must satisfy (i)-(iii). We have

$$
U_{1}\left(f_{0}, g_{0}\right) U_{1}(f, g)=U_{1}(f, g) U_{1}\left(f_{0}, g_{0}\right) e^{i\left\langle f, g_{0}\right\rangle-i\left\langle f_{0}, g\right\rangle} .
$$

If $f_{0} \in W_{\varphi}, g_{0} \in W_{\pi}$ this gives

$$
U\left(f_{0}, g_{0}\right) X(f, g) U(f, g)=X(f, g) U(f, g) U\left(f_{0}, g_{0}\right) e^{i\left\langle f, g_{0}\right\rangle-i\left\langle f_{0}, g\right\rangle}
$$

and thus

$$
\begin{aligned}
U\left(f_{0}, g_{0}\right) X(f, g) & =X(f, g) U(f, g) U\left(f_{0}, g_{0}\right) U(f, g)^{-1} e^{i\left\langle f, g_{0}\right\rangle-i\left\langle f_{0}, g\right\rangle} \\
& =X(f, g) U\left(f_{0}, g_{0}\right) .
\end{aligned}
$$

Thus

$$
X(f, g) \in\left\{U(f, g) ; f \in W_{\phi}, g \in W_{\pi}\right\}^{\prime}=\left\{U(f, g) ; f \in V_{\phi}, g \in V_{\pi}\right\}^{\prime}
$$

which proves (iv). The Weyl commutation relation

$$
U_{1}\left(f_{1}, g_{1}\right) U_{1}\left(f_{2}, g_{2}\right)=U_{1}\left(f_{1}+f_{2}, g_{1}+g_{2}\right) e^{-i\left\langle f_{2}, g_{1}\right\rangle}
$$

gives

$$
\begin{array}{r}
X\left(f_{1}, g_{1}\right) U\left(f_{1}, g_{1}\right) X\left(f_{2}, g_{2}\right) U\left(f_{2}, g_{2}\right)=X\left(f_{1}+f_{2}, g_{1}+g_{2}\right) \\
U\left(f_{1}+f_{2}, g_{1}+g_{2}\right) e^{-i\left\langle f_{2}, g_{1}\right\rangle}
\end{array}
$$

(v) now follows from (iv) and the Weyl commutation relation for $U(f, g)$.

If $U_{1}(f, g)$ is unitarily equivalent to $U(f, g)$ we have

$$
U_{1}(f, g)=V U(f, g) V^{-1}
$$

for some $V$. It follows that

$$
U(f, g)=V U(f, g) V^{-1}, f \in W_{\phi}, g \in W_{\pi}
$$

and thus

$$
V \in\left\{U(f, g) ; f \in W_{\phi}, g \in W_{\pi}\right\}^{\prime}=\left\{U(f, g) ; f \in V_{\phi}, g \in V_{\pi}\right\}^{\prime}
$$

which implies that $U_{1}(f, g)=U(f, g)$ for all $f \in V_{\phi}, g \in V_{\pi}$. Q.E.D.

If in the above theorem we drop the hypothesis that

$$
\left\{U(f, g) ; f \in W_{\phi}, g \in W_{\pi}\right\}^{\prime \prime}=\left\{U(f, g) ; f \in V_{\phi}, g \in V_{\pi}\right\}^{\prime \prime}
$$

then condition (iv) becomes $X(f, g) \in\left\{U(f, g), f \in W_{\phi}, g \in W_{\pi}\right\}^{\prime}$, (v) becomes

$$
X\left(f_{1}, g_{1}\right) U\left(f_{1}, g_{1}\right) X\left(f_{2}, g_{2}\right) U\left(f_{1}, g_{1}\right)^{-1}=X\left(f_{1}+f_{2}, g_{1}+g_{2}\right),
$$


and $U_{1}(f, g)$ unitarily equivalent to $U(f, g)$ need not imply that $X(f, g)=1$. It should be noted that this hypothesis is implied by but is not equivalent to the condition that $W_{\phi}, W_{\pi}$ are dense in $V_{\phi}, V_{\pi}$ in the topologies $\tau_{\phi}, \tau_{\pi}$ induced by the representation $U(f, g)$.

In order to illustrate more clearly the pathological behaviour of representations like the $U_{1}(f, g)$ constructed above, let $V_{\phi}=V_{\pi}=V$ where $V$ is a real Hilbert space and let $U(f, g)$ be the Fock representation $U_{F}(f, g)$. Let $W_{\phi}=W_{\pi}=W$ be the algebraic span of an orthonormal basis for $V$, and consider the irreducible representations of the CCRs over $V$ given by

$$
U\left(f, g ; T_{\phi}, T_{\pi}\right)=e^{i T_{\phi}(f)} e^{i T_{\pi}(g)} U_{F}(f, g)
$$

where $T_{\phi}, T_{\pi}$ are linear maps from $V$ into $R$ satisfying $T_{\phi}=T_{\pi}=0$ when restricted to $W$. Since $\left\{U_{F}(f, g) ; f, g \in W\right\}^{\prime \prime}=\boldsymbol{B}(H)$ it follows from Theorem 6.1 that $U\left(f, g ; S_{\phi}, S_{\pi}\right)$ is unitarily equivalent to $U\left(f, g ; T_{\phi}, T_{\pi}\right)$ if and only if $S_{\phi}=T_{\phi}$ and $S_{\pi}=T_{\pi}$. If $V$ is countably infinite dimensional as a Hilbert space, then the algebraic dimension of $V$ is $c$, and there are $c^{c}$ different linear maps $T_{\phi}, T_{\pi}$ which vanish on $W$. Thus we have $c^{c}$ many inequivalent irreducible representations of the CCRs over the real Hilbert space $V$ which coincide with the Fock representation when restricted to the algebraic span $W$ of an orthonormal basis for $V$ (clearly this statement holds for any subspace $W$ whose algebraic codimension is $c$ ). As a further illustration of the undesirable behaviour of these representations, we give an alternate proof that $U\left(f, g ; T_{\phi}, T_{\pi}\right)$ unitarily equivalent to $U_{F}(f, g)$ implies that $T_{\phi}=T_{\pi}=0$. Since $U_{F}(f, g)$ is strongly continuous in $f$ and $g$ in the norm topology, we have for all $f, g \in V$, $U_{F}(f, g)=$ strong $\lim U_{F}\left(f_{\alpha}, g_{\alpha}\right)$ where $f_{\alpha}, g_{\alpha} \in W$ and $\left\|f_{\alpha}-f\right\|,\left\|g_{\alpha}-g\right\| \rightarrow 0$. But $U\left(f, g ; T_{\phi}, T_{\pi}\right)$ unitarily equivalent to $U_{F}(f, g)$ implies that $U(f, g$; $\left.T_{\phi}, T_{\pi}\right)=$ strong $\lim U\left(f_{\alpha}, g_{\alpha} ; T_{\phi}, T_{\pi}\right)$. Since $U\left(f_{\alpha}, g_{\alpha} ; T_{\phi}, T_{\pi}\right)=U_{F}\left(f_{\alpha}, g_{\alpha}\right)$ if $f_{\alpha}, g_{\alpha} \in W$, it follows that $U\left(f, g ; T_{\phi}, T_{\pi}\right)=U_{F}(f, g)$ for all $f, g \in V$, and thus $T_{\phi}=T_{\pi}=0$. It follows from this argument that if we take the representation $U\left(f, g ; T_{\phi}, T_{\pi}\right)$, restrict it to $W$, and then extend it by continuity according to Theorem 6.1, we obtain the Fock representation rather than the original representation $U\left(f, g ; T_{\phi}, T_{\pi}\right)$. Another undesirable property of these representations is the behaviour of the total number operator which exists for some orthonormal bases but not for others (see [6] for a detailed discussion of this).

We now construct some more examples.

Lemma 7.2. Let $U(f, g)$ be a representation of the CCRs over $V_{\phi} \times V_{\pi}$. Let $W_{\phi}$ be a subspace of $V_{\phi}$. Then there exists a representation $U_{1}(f, g)$ of the CCRs over $V_{\phi} \times V_{\pi}$ such that $W_{\phi}$ is closed in the topology $\tau_{\phi}$ induced on $V_{\phi}$ by $U_{1}(f, g)$. 
Proof. Let $H$ be the Hilbert space on which the representation $U(f, g)$ is defined. Let

$$
\begin{aligned}
H_{1} & =H \otimes H, & & \\
U_{1}(f) & =U(f) \otimes 1, & & f \in W_{\phi}, \\
V_{1}(g) & =V(g) \otimes 1, & & g \in V_{\pi} .
\end{aligned}
$$

Let $\left\{h_{\alpha}, \alpha \in A\right\}$ be an algebraic basis for $W_{\phi}$ and extend it to an algebraic basis $\left\{h_{\alpha}, \alpha \in A ; h_{\beta}^{\prime}, \beta \in B\right\}$ for $V_{\phi}$. We extend the definition of $U_{1}(f)$ to $V_{\phi}$ by

$$
U_{1}\left(s h_{\beta}^{\prime}\right)=U\left(s h_{\beta}^{\prime}\right) \otimes U\left(s h_{\beta}^{\prime}\right), \quad \beta \in B
$$

If $f \notin W_{\phi}$ we have $f=f^{\prime}+f^{\prime \prime}$ where $f^{\prime} \in W_{\phi}, f^{\prime \prime} \in V-W_{\phi}$ and $f^{\prime \prime} \neq 0$. Then $U_{1}(f)=U\left(f^{\prime}+f^{\prime \prime}\right) \otimes U\left(f^{\prime \prime}\right) \notin \boldsymbol{B}(H) \otimes 1$. Thus $f \notin W_{\phi}$ implies that $U_{1}(f)$ can not be the strong limit of a net $U_{1}\left(f_{\alpha}\right), f_{\alpha} \in W_{\phi}$. But if $f$ is in the closure of $W_{\phi}$, it follows from the definition of the topology $\tau_{\phi}$ that there exist $f_{\alpha} \in W_{\phi}$ such that $U_{1}(f)=$ strong $\lim U_{1}\left(f_{\alpha}\right)$. Thus $W_{\phi}$ is closed. Q.E.D.

Lemma 7.3. Let $U(f, g)$ be a representation of the CCRs over $V_{\phi} \times V_{\pi}$, and let $W_{\phi}$ be a proper subspace of $V_{\phi}$. Then there exists a representation $U_{1}(f, g)$ of the CCRs over $V_{\phi} \times V_{\pi}$ such that

$$
\left\{U_{1}(f, g), f \in W_{\phi}, g \in V_{\pi}\right\}^{\prime \prime} \neq\left\{U_{1}(f, g), f \in V_{\phi}, g \in V_{\pi}\right\}^{\prime \prime} .
$$

Proof. Repeat the construction in the proof of Lemma 7.2. Then $U_{1}(f) \in \boldsymbol{B}(H) \otimes 1$ if and only if $f \in W_{\phi}$. Q.E.D.

The undesirable behaviour of the above examples seems to be essentially the fact that, given $V_{\phi}, V_{\pi}$ and proper subspaces $W_{\phi}, W_{\pi}$ which are "sufficiently large" in the sense that some representations of the CCRs over $V_{\phi} \times V_{\pi}$ are determined by continuity by their restriction to $W_{\phi} \times W_{\pi}$ (see Theorem 4.2), there exist representations which are not determined by their restriction to $W_{\phi} \times W_{\pi}$. Now one might be inclined to consider a representation of the CCRs pathological if it differed from the Fock representation but coincided with the Fock representation on some subspace $W$ which is dense in $V$ in the norm topology on $V$. However consider the following example. Let $V=L^{2}\left(R^{3}\right) \cap L^{1}\left(R^{3}\right)$. Let $U_{F}(f, g)$ be the Fock representation of the CCRs over $V$ on the Hilbert space $H_{F}$. Let

$$
\tilde{f}(0)=\int f(\boldsymbol{x}) d \boldsymbol{x} .
$$

Then the irreducible representation of the CCRs defined by

$$
U_{\alpha, \beta}(f, g)=U_{F}(f, g) e^{i \alpha \tilde{f}(0)+i \beta \tilde{g}(0)}
$$

2 Commun. math Phys, Vol. 17 
describes the ground state of an infinite nonrelativistic free Bose gas at a finite density [2]. Now, as was pointed out to us by H. Araki, this representation coincides with the Fock representation when restricted to the subspace

$$
W=\{f \in V: \tilde{f}(0)=0\}
$$

which is obviously dense in $V$ in the norm topology. (Incidentally, Theorem 7.1 now provides an alternate proof that the parameters $\alpha, \beta$ label unitarily inequivalent representations $U_{\alpha, \beta}(f, g)$.)

We now propose a somewhat milder restriction which seems reasonable from the viewpoint of physical applications. In quantum field theory one typically constructs a representation of the CCRs over some suitably restricted space of test functions (e.g. the Schwartz space $\mathscr{D}$ of $C^{\infty}$ functions on $R^{n}$ with compact support). One can then extend the representation by continuity to a larger space $V$ of test functions according to Theorem 6.4. For example, the representation $U_{\alpha, \beta}(f, g)$ given by Eq. (7.1) can obviously be extended by continuity from $\mathscr{D}$ to $L^{1}\left(R^{3}\right) \cap L^{2}\left(R^{3}\right)[2]$. We propose

Definition 7.4. Let $C_{\phi}, C_{\pi}$ be classes of subspaces of $V_{\phi}, V_{\pi}$. We say that a representation $U(f, g)$ of the CCRs over $V_{\phi} \times V_{\pi}$ is smooth relative to the classes $C_{\phi}, C_{\pi}$ if $W_{\phi} \in C_{\phi}, W_{\pi} \in C_{\pi}$ implies that $W_{\phi}, W_{\pi}$ are dense in the topologies $\tau_{\phi}, \tau_{\pi}$ induced by the representation $U(f, g)$.

For physical applications, consider the situation where $\mathscr{D} \subset V \subset L^{2}\left(R^{n}\right)$ where $\mathscr{D}$ is the Schwartz space of $C^{\infty}$ functions on $R^{n}$ with compact support. We would then take $C_{\phi}=C_{\pi}$ to be the set of all subspaces $W \subset V$ such that $W \cap \mathscr{D}$ is dense in $\mathscr{D}$ in the Schwartz topology $\tau_{S}$ on $\mathscr{D}$. Since $f_{\alpha} \rightarrow f\left(\tau_{S}\right), f, f_{\alpha} \in \mathscr{D}$ implies that $\tilde{f}_{\alpha}(0) \rightarrow \tilde{f}(0)$ and $\left\|f_{\alpha}-f\right\|_{2} \rightarrow 0$ it follows that the representations $U_{\alpha \beta}(f, g)$ are smooth in this sense.

The following two lemmas are completely trivial, but they illustrate to what extent the above condition eliminates the pathologies discussed above.

Lemma 7.5. Let $U_{1}(f, g), U_{2}(f, g)$ be representations of the CCRs over $V$ which are smooth relative to the class $C$. Then $U_{1}(f, g)=U_{2}(f, g)$, $f \in W_{\phi}, g \in W_{\pi}$ where $W_{\phi}, W_{\pi} \in C$ implies that $U_{1}(f, g)=U_{2}(f, g)$ for all $f \in V_{\phi}, g \in V_{\pi}$.

Proof. Follows immediately from Theorem 6.4. Q.E.D.

Lemma 7.6. Let $U(f, g)$ be a representation of the CCRs over $V$ which is smooth relative to the class $C$. Then $W_{\phi}, W_{\pi} \in C$ implies that

$$
\left\{U(f, g), f \in W_{\phi}, g \in W_{\pi}\right\}^{\prime \prime}=\left\{U(f, g), f \in V_{\phi}, g \in V_{\pi}\right\}^{\prime \prime} .
$$


Proof. By assumption $W_{\phi}, W_{\pi}$ are dense in $V_{\phi}, V_{\pi}$ in the topologies $\tau_{\phi}, \tau_{\pi}$ induced by the representation $U(f, g)$. It then follows from the definition of $\tau_{\phi}, \tau_{\pi}$ that we have $U(f, g)=$ strong $\lim U\left(f_{\alpha}, g_{\alpha}\right)$ where $f_{\alpha} \in W_{\phi}, g_{\alpha} \in W_{\pi}$ for all $f \in V_{\phi}, g \in V_{\pi}$. Q.E.D.

It is not clear to us whether or not the concept of a smooth representation will turn out to be useful.

Note added in proof. $\mathrm{H}$. Araki has provided an example of representations $U_{t}(f, g)$, $i=1,2$ and a subspace $W$ which is dense relative to the induced topologies $\tau_{\phi i}$, but which is not dense relative to the topology $\tau_{\phi}$ induced by $U_{1}(f, g) \oplus U_{2}(f, g)$.

\section{Representations of Topological Groups}

It seems likely that the above discussion of the continuity properties of the representations of the CCRs is relevant to the representations of topological groups which are not locally compact. By a continuous representation of a topological group $(G, \tau)$ we mean a representation of $G$ by operators $T(g)$ in a Hilbert space $H$ such that the map $g \rightarrow T(g)$ is strongly continuous. However in general a given representation $T(g)$ will also be continuous in strictly coarser topologies for $G$. One could define the topology $\tau_{T}$ induced on $G$ by the representation $T(g)$ to be the weakest topology such that the map $g \rightarrow T(g)$ is continuous. Whether or not $\left(G, \tau_{T}\right)$ is the desired topological group depends on the situation (this definition is not suitable for the CCRs). In Section 7 we constructed some representations of the CCRs which exhibited "undesirable" continuity properties. Similarly if $G$ is a topological group which is not locally compact (e.g. an infinite dimensional Lie group) then in general there will exist representations of $G$ which are not determined by continuity by their restriction to a subgroup $G_{0}$ which is "sufficiently large" in the sense that some representations of $G$ are determined by their restriction to $G_{0}$. By analogy with Definition 7.4 one could define a representation of $G$ to be smooth relative to the class $C$ of subgroups of $G$ if the representation is determined by its restriction to every $G_{0} \in C$.

Acknowledgements. We would like to thank D. Gregory, J. R. Klauder, and J. McKenna for helpful comments, and H. Araki, G. Elliott and G. Hegerfeldt for pointing out some errors in an earlier version of this paper. We are especially indebted to $\mathrm{H}$. Araki for several stimulating discussions. This work was essentially completed while the author was a member of the Summer Research Institute of the Canadian Mathematical Congress at McGill University.

\section{References}

1. Araki, H.: J. Math. Phys. 1, 492 (1960).

2. - Woods, E. J.: J. Math. Phys. 4, 637 (1963).

3. - - Publ. RIMS, Kyoto Univ. Ser. A, 2, 157 (1966). 
4. - - Topologies on Test Function Spaces induced by Representations of the Canonical Commutation Relations.

5. Bourbaki, N.: Topologie Générale, 3rd Ed. Paris: Hermann 1960.

6. Chaiken, J. M.: Ann. Phys. (N. Y.) 42, 23 (1967).

7. - Commun. Math. Phys. 8, 164 (1968).

8. Cook, J. M.: Trans. Am. Math. Soc. 74, 222 (1953).

9. Friedrichs, K. O.: Mathematical aspects of the quantum theory of fields. New York: Interscience 1953.

10. Gårding, L., and Wightman, A. S.: Proc. Natl. Acad. Sci. U.S. 40, 622 (1954).

11. Gel'fand, I. M., Vilenkin, N. Ya.: Generalized functions, Vol. 4. New York: Academic Press 1964.

12. Hegerfeldt, G. C., Klauder, J. R.: Metrics on test function spaces for canonical field operators. Commun. Math. Phys. 16, 329-346 (1970).

13. Kelley, J. L.: General topology. New York: van Nostrand 1955.

14. Klauder, J. R., McKenna, J., Woods, E. J.: J. Math. Phys. 7, 822 (1966).

15. Lew, J. S.: unpublished thesis, Princeton, 1960.

16. von Neumann, J.: Math. Ann. 104, 570 (1931).

17. - Compos. Math. 6, 1 (1938).

18. Reed, M.: On the self-adjointness of quantum fields and hamiltonians (Stanford Ph. D. thesis, 1968).

19. - A Gårding domain for quantum fields. Commun. Math. Phys. 14, 336-346 (1969).

20. Segal, I. E.: Trans. Am. Math. Soc. 88, 12 (1958).

21. - Mathematical problems of relativistic physics. Providence, R. I.; Am. Math. Soc. 1963.

22. Streit, L.: Commun. Math. Phys. 4, 22 (1967).

E. J. Woods

Department of Mathematics

Queen's University

Kingston, Ontario, Canada 